Integrating Metrics into a Large-Scale

Software Development Enviroment

Sallie Henry and John Lewis

TR 89-1 


\title{
Integrating Metrics into a Large-Scale Software Development Environment
}

\author{
Sallie Henry and John Lewis
Computer Science Department, Virginia Polytechnic Institute and State University, Blacksburg, Virginia
}

\begin{abstract}
Software metrics have been shown to be indicators of software complexity. These complexity indicators identify early can be rewritten and/or thoroware, which if identified the integration of metric/or thoroughly tested. However, faces practical resistance use into commercial situations strictions. This paper presents budget and deadline reduces a nondisruptive methods an experiment that intro. a large-scale commercial software integrating metrics into ment.
\end{abstract}

\section{INTRODUCTION}

A major goal of software producers is to develop relidevelopment is code. However, given that software constraints, the critersarily guided by budget and time is often that it satisfy the internal software acceptance Unfortunately, correct requirements of specific tests. plan does not guarantee software according to the test ability or other quality factors software in terms of reli-

The key to integrating quators [1].

from the relationship beting quality into software stems rors. As the complexity increases, the code becomes level a piece of software is more likely to contain diffcult to understand and ing errors must be modified, defects. Code containnontrivial since the code is diff that maintenance is compound the problem further, it to comprehend. To programs cannot be made more, it has been shown that changing their code. In fact more maintainable by simply lished that maintenan fact, Belady and Lehman establevel of complexing activities tend to increase the likely to be required [2]

into software the effort to instill quality characteristics opment process. have been proposed to software engineering techniques

\footnotetext{
Address correspond

Department, Virginia Polytechnic Institute an, Computer Science Blacksburg, VA 24061.
}

ware, and consequently increase reliability and other are difficult to Linfortunately, most of these techniques to be an expected conse and enforce. If maintainability is ways to measure it must bece of software development, development cycle. Thereforme an integral part of the evaluation of solwerefore, a subjective, quantitative

Software software complexity is needed.

have been shown then defined and used correctly, plexity [3]. Metrics are guandicators of software comware design or code gre quantitative evaluations of softcontribute to the softwared on some set of criteria that ysis results were required complexity. If metric analances, the development to conform to quality tolermised.

Software complexify problem of low-quality metrics will not solve the entire shown that metrics can predict. However, studies have can indicate where a redesign error proneness [4] and These aspects of softedesign effort is beneficial [5]. for concentrating specife quality represent good points

This paper describes a methement efforts.

ware metrics to integrate some quality fhat uses softscale software. The methodology is factors into largecommercial environment of a majo implemented in a as a means to complement a major software producer process. This cooperarion their software development developers and academion between commercial software of metric research remicians is essential for validation past projects concerning. This research complements large-scale software metrics [7], tool development [6], ships with other software developm, and working relation-

One requirement of the projopment organizations [8]. of a tool and associated methodology the development unobtrusively to current softodology that would operate matically generated quantitative production, that autoprovide threshold values for metrics, and that would

To test the values for metric interpretation. ogy is used to analyze of the model, the methodoldevelopment release point of code frozen in a mid- 